\title{
ANALYSIS OF THE EFFECT OF TWO-WHEELER LANE-SHARING BEHAVIOR ON MACROSCOPIC TRAFFIC FLOW MODELING
}

\author{
Mallikarjuna Chunchu, Bhavathrathan Bhattiyil Kuzhiyamkunnath \\ Dept of Civil Engineering, Indian Institute of Technology Guwahati, Assam, India
}

Submitted 20 August 2012; resubmitted 25 February 2013; accepted 9 April 2013

\begin{abstract}
Small size motorized vehicles such as the motorized two-wheelers are predominantly present in the traffic observed in the developing countries. Presence of these smaller vehicles invalidates the concept of lane discipline commonly observed in the homogeneous traffic streams. In this paper an attempt has been made to understand the Motorized Two-Wheeler (MTW) lane-sharing behavior and its effects on traffic flow modeling. The objectives of this attempt are to analyze the lane-sharing behavior of MTWs at microscopic and macroscopic level, and to propose a methodology to incorporate this behavior in the macroscopic modeling concepts. Effect of the MTW lane-sharing behavior on the microscopic and macroscopic traffic characteristics and the corresponding models is discussed. A plausible way to incorporate this behavior, specifically in macroscopic modeling, is discussed. From a specific analysis based on VISSIM (version 5.20) it has been observed that the MTW lane-sharing behavior significantly influence the equilibrium speed-density relationships which are the key inputs to the macroscopic traffic flow models. Density diminishing factor is found to be significantly influenced by the proportion of MTWs present in the traffic stream. It has been observed that the lane-sharing behavior starts taking place when the average stream speed is less than $65 \mathrm{~km} / \mathrm{hr}$, for the proportions of MTWs in between 25 to $60 \%$. For further higher MTW proportions lane-sharing has been observed even in free flow conditions.
\end{abstract}

Keywords: motorized two-wheeler; heterogeneous traffic; macroscopic model; no-lane discipline; density diminishing factor.

Reference to this paper should be made as follows: Chunchu, M.; Kuzhiyamkunnath, B. B. 2014. Analysis of the effect of two-wheeler lane-sharing behavior on macroscopic traffic flow modeling, Transport 29(2): 146-153.

http://dx.doi.org/10.3846/16484142.2014.928788

\section{Introduction}

Heterogeneous traffic observed in the developing countries like India is quite complex to model due to numerous reasons. Some of the important reasons are the traffic composition and the absence of lane discipline even when the lane markings are present (lane width varies between 3.5 to $3.75 \mathrm{~m}$ ). The latter reason cannot be attributed to the indiscipline of the drivers and the actual reason behind the absence of lane discipline may be the presence of smaller vehicles in the traffic stream. In general the cars used in India are also smaller in size when compared to the cars used in the developed countries. In these conditions on $3.75 \mathrm{~m}$ wide single lane there can be overtaking/passing operations involving the Motorized Two-Wheelers (MTWs) and the other vehicles. When the road width is 7.5 to $10.5 \mathrm{~m}$ (two-lane or three-lane roads), there are numerous ways in which a faster and smaller following vehicle overtakes/passes the leading vehicle or vice versa.

In the absence of lane discipline, it is difficult to apply the conventional microscopic or the macroscopic traffic variables to study and model the heterogeneous traffic. If these variables are used in the modeling without understanding the ramifications, the results may misguide the modeler. In this scenario it is necessary to understand and incorporate the absence of lane discipline into the modeling process.

In this study an attempt is made to understand and incorporate the absence of lane discipline into the modeling process. The absence of lane discipline is assumed to be resulting from the presence of the smaller vehicles on the sides of the other vehicles in the same lane and it is termed as MTW lane-sharing. The impact of the lane-sharing of the MTWs is analyzed at microscopic

Corresponding author: Mallikarjuna Chunchu

E-mails: c.mallikarjuna@iitg.ernet.in; chmallikarjuna@yahoo.com 
and macroscopic levels. A factor, termed as the density diminishing factor, has been formulated to incorporate the lane-sharing behavior into the macroscopic models. Lane-sharing has been simulated using the VISSIM (version 5.20) software, which allows overtaking/passing in the same lane. The results from the VISSIM, in terms of speed-density relationships, have been used in knowing the effect of the lane-sharing behavior.

\section{Literature Review}

Not much work has been done in the past that deals with the operations of smaller vehicles such as the MTWs and motorized three wheelers which are present in significant numbers in most of the developing countries. This section presents the relevant literature focusing mostly on the macroscopic models.

Many researchers in the past have modified the Lighthill, Whitham, and Richards (LWR) model to study the homogeneous traffic stream with the heterogeneous drivers travelling at different desired speeds (Wong, Wong 2002; Gupta, Katiyar 2007; Ngoduy, Liu 2007; Logghe, Immers 2008). Wong and Wong (2002) have considered the heterogeneity of traffic with reference to different speeds that each group of vehicles opts. This extension is based on the surmise that the choice of speed by a particular user class is not only affected by the presence of this user class, but also by all other user classes on the highway. They related the speed of the each user group as a function of the total density. In a similar work, Ngoduy and Liu (2007) have identified the relevance of the traffic regime in this formulation. They proposed that a particular class speed will very well be a function of the total stream density, but only when the stream density values are less than the critical density. In other words, the speed differences among the classes happen only in the unrestricted traffic regime. In the congested regime all the vehicles move with the same speed. This formulation appears more likely as this would mean that the vehicles will overtake only while moving freely, and not when moving under the congested conditions. Gupta and Katiyar (2007) have developed a continuum model with anisotropic nature and can model the vehicles opting for various speed levels. They have also assumed that the speeds opted by a particular vehicle class is affected by the presence of all user classes on that location.

Logghe and Immers (2008) have proposed a new model where the slow moving vehicles hinder the movement of the capable fast movers. Three regimes or phases can be distinguished according to the class densities: the free-flow regime, the semi-congestion regime, and the congestion regime. In the free flow regime, vehicles move with their free flow speed without disrupting each other. In semi-congested regime the fast vehicles are in congested region and would reduce their speed but not less than speed of the slow vehicles, such that the hindrance from the slower vehicles cause the speed reduction in capable fast movers. Needless to say, slow vehicles travelled with their free flow speed. In congested region, all vehicles move with same speed, as in the case of Ngoduy and Liu (2007).

Some of the researchers have worked on the analysis of MTW behavior near intersections (Rongviriyapanich, Suppattrakul 2005; Lee et al. 2009; Haque et al. 2008; Su et al. 2006). Minh et al. (2005) have studied the operations of the MTWs on mid-block sections. To incorporate the MTW behavior, specifically when approaching the signalized intersections, Powell (2000) made some changes to the first order macroscopic traffic flow model. He has attempted to find the number of MTWs that percolate through the queue and reach the front end of the queue.

Tang et al. (2010) have proposed a dynamic model for the heterogeneous traffic stream consisting of cars, bicycles, and pedestrians. All these three elements of the traffic stream move on separate lanes and the movement of each of these elements is influenced by the traffic stream moving on the adjacent lane. The friction effects at microscopic level are translated into the corresponding macroscopic variables which are the basis for the dynamic model. Tang et al. (2011) have proposed another macroscopic model considering the honk effects on the movement of pedestrian and cycle. A detailed review of the macroscopic models and their evolution in the context of heterogeneous traffic flow modeling can be found in Bhavathrathan and Mallikarjuna (2012).

Microscopic simulation models allow explicit rule based vehicle movements and are convenient for modeling the MTWs and the other smaller vehicles. Many researchers have developed microscopic simulation models incorporating the MTWs behavior and in most of these models the vehicles are updated based on the explicit interactions between the vehicles (Khan, Maini 1999; Meng et al. 2007; Lee et al. 2009; Mallikarjuna, Ramachandra Rao 2009; Lan et al. 2010). Understanding and modeling the explicit interactions among the vehicles require huge amount of data and even if they are modeled at microscopic level it is difficult to validate the model using the microscopic observations.

\section{Motivation and the Objectives}

When the smaller vehicles are predominantly present in the traffic stream there is a competition among the vehicles to utilize the available road space. This competition compels the MTW riders to utilize the space available alongside the four-wheelers, making the values of the flow and the density indefinite. Thus, for a model to be useful in simulating the Indian traffic conditions, it inevitably has to incorporate the side-by-side movement of the MTWs and the four-wheelers.

Specifically in a developing country like India, utilization of the space adjacent to the cars cannot be regarded as illegitimate on the parts of MTWs as they are not violating any established guidelines. In this context, where already the supply is on lower side, a law could not be framed to stop this as such a regulation would result in the under utilization of the urban road space. Alternatively efforts must be put in to consider the effect of 
this phenomenon while planning the infrastructure. In the near future the traffic flow models will be more and more employed in the decision making process hence it is important that this phenomenon is considered in such models. The phenomenon of lane-sharing is explained in the following paragraphs.

Fig. 1a shows a possible scenario in the heterogeneous traffic stream moving under free flow conditions (minimal interaction between the vehicles). As can be seen from the figure the MTWs do not exhibit the side-by-side movement hence the existing macroscopic models are capable of representing this scenario. Fig. $1 \mathrm{~b}$ illustrates the heterogeneous traffic stream at a slightly higher density. The distance between the four-wheelers reduces, and the MTWs start moving side-by-side to each other.

At even higher densities, the MTWs opt out from their previous positions, and start occupying the available space on the sides of the leading four-wheelers, as shown in Fig. 1c. On the urban roads in India the peak hour traffic conditions will mostly be as shown in Figs 1c and $1 \mathrm{~d}$. The MTWs move on the sides of four-wheelers for considerable amount of time, and the utility of this empty space is a function of the density and the number of MTWs present in the traffic stream. Thus, models that do not account for the property illustrated in Figs 1c and $1 \mathrm{~d}$ cannot be used as-such to represent the Indian urban traffic conditions.

Theoretically, the macroscopic models are the expanded forms of the microscopic models. The microscopic models adopt parameters like headway and vehicle speed, which are defined based on the vehicle trajectories of the time-space diagram. In the macroscopic models the time and the space headways are translated in to the macroscopic variables flow, and the density, respectively. Inverse of the average time headway corresponds to the flow and the inverse of the average space headway corresponds to the density.

Trajectories of vehicles, commonly observed in homogeneous traffic conditions, are shown in Fig. 2a. The difference in the time-space diagrams of the heterogeneous traffic without and with the MTW lane-sharing is shown in Figs $2 b$ and $2 c$, respectively. These figures represent the traffic movement observed on a single

a)

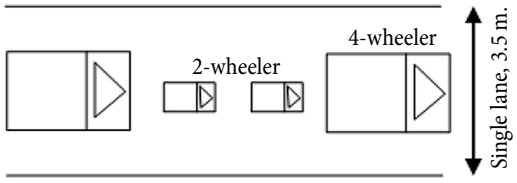

c)

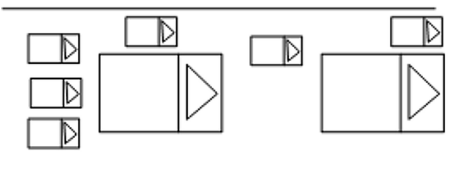

a)

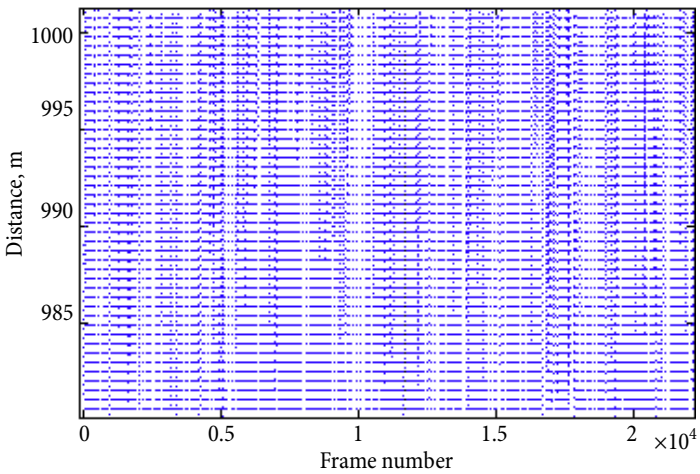

b)

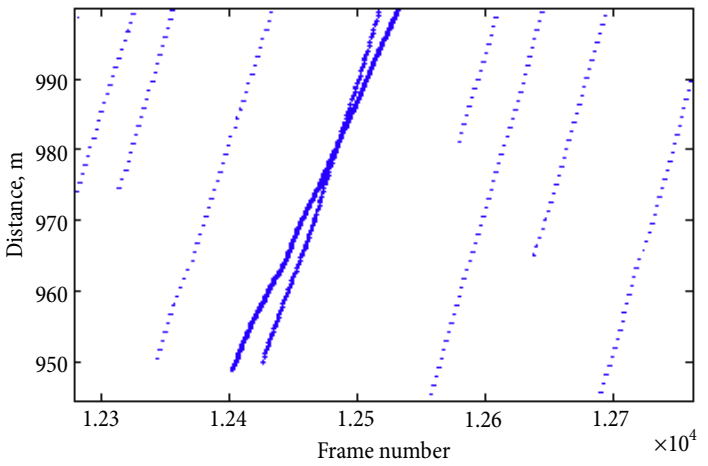

c)

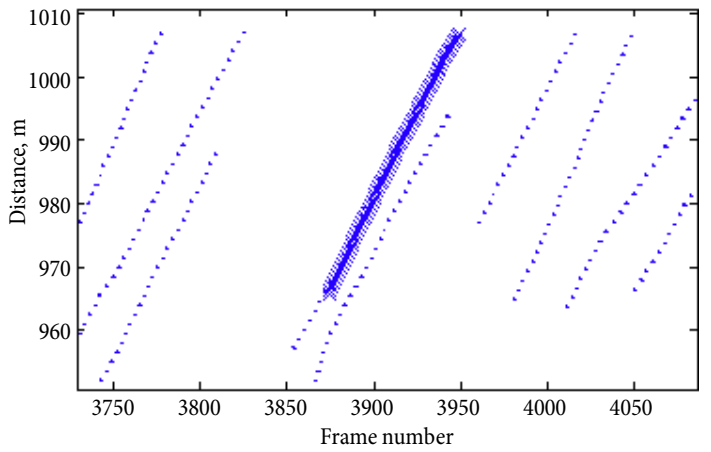

Fig. 2. Snap shots of the vehicular trajectories observed over a single lane of a three-lane wide road, carrying the heterogeneous traffic: a - trajectories of the vehicles moving on a single lane; $b$ - observed overtaking operation on a single lane; $\mathrm{c}$ - observed side-by-side movement on a single lane (dotted and the crossed lines representing two trajectories)

b)

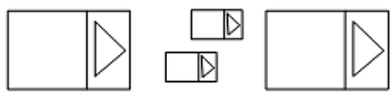

d)

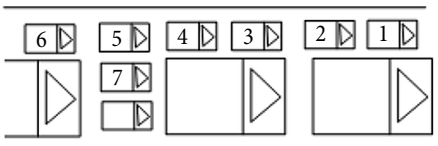

Fig. 1. MTW and four-wheeler interaction assumed in the conventional models, equivalent to the heterogeneous free flow traffic (a); MTWs with the side-by-side movement at higher densities (b); MTWs occupying the sides of four-wheelers at even higher densities (c); peak-hour conditions or jam conditions (d) 
lane of a three-lane wide road. Trajectory data have been extracted from the video film collected on a three lane road located on the outskirts of Delhi using the video image processing software - TRAZER' (TRaffic AnalyZer and EnumeratoR). Fig. $2 \mathrm{~b}$ represents the traffic conditions where the MTWs quickly pass the other vehicles and Fig. $2 c$ represents the traffic where MTWs move on the sides of the other vehicles for considerable amount of time.

Trajectories in Fig $2 \mathrm{~b}$ show that none of the vehicles are moving side-by-side (along the length of the single lane road) except during the process of overtaking. Even this is a peculiar feature observed only in the absence of lane discipline. Fig. $2 \mathrm{c}$ illustrates the trajectories of MTWs coinciding with that of the other vehicles, in other words the MTWs and the other vehicles are traveling side-by-side for about $40 \mathrm{~m}$ distance. From Fig. 2c the density observed at frame number 3900 is two vehicles over a length of $60 \mathrm{~m}$ road length. Even though there are three vehicles present over this road stretch due to the side-by-side movement the density value turns out to be two vehicles over the observed road length. The effect of the lane-sharing behavior on the density values is further explained in the following sections.

\section{The Concept of Diminishing Density}

It is known that the vehicular speeds are influenced by the longitudinal space headways. When dealing with the traffic at macroscopic level it may be said that the average speed of the traffic stream is influenced by density. When MTWs are sharing the lane, longitudinal headways may be correlated to the lane-sharing behavior in addition to the density. In these conditions, even when the observed densities are high (the actual number of vehicles seen on the road) space headways available for the vehicles may not be that low. Considering this, and also considering the importance of the speed-density relationship in the macroscopic traffic flow models, it is hereby suggested that the density is the major parameter that should be adjusted.

Del Castillo and Benítez (1995) have proposed several forms of the speed-density relationships. All these relationships have the following functional form in common:

$$
V=V_{f} \cdot(1-f(\lambda)),
$$

where: $V_{f}$ is the free-flow speed of the traffic stream; $f(\cdot)$ is the generating function and its argument; $\lambda$ is equivalent spacing:

$$
\lambda=\frac{\left|C_{j}\right|}{V_{f}} \cdot\left(\frac{H}{H_{j}}-1\right),
$$

where: $C_{j}$ is the kinematic wave speed at jam density; $H_{j}$ is the jam spacing; $H$ is the average spacing, the reciprocal of density.

It is thus evident that the functional form of the speed-density relationship is based mainly on the longitudinal spacing between the vehicles. Therefore, to in- corporate the lane-sharing behavior, the observed values of density on the Indian roads should be corrected before being used in the speed-density relationships, and subsequently the modified speed-density relationships are to be given as the input into the existing macroscopic models. For this purpose, a concept that deals with the diminishing density is proposed in this study.

\subsection{The Density Diminishing Factor}

Considering the roadway shown in Fig. 1d, one may find the density as eleven vehicles per unit length, just by counting. However, as explained earlier, the existence of MTWs numbered one to seven cannot be incorporated in the speed-density equation. In the absence of side-by-side movement, the density would have been four vehicles per unit length. Thus, in the perspective of speed-density relationship, any observed density will thus have an equivalent suitable value, which will be lower in magnitude. At the same time the density value cannot be considered as four vehicles (the side-by-side movement has certain effect such as the lateral friction on the speed of the vehicles) and it may be anywhere in between 4 and 11. To find this equivalent value, the observed density will have to be diminished by a factor. Calling this as the density diminishing factor, and denoting it with $\Phi$ :

$$
\rho^{*}=\Phi \cdot \rho,
$$

where: $\Phi$ is density diminishing factor, the range is $0<\Phi \leq 1 ; \rho$ is the total observed density of the traffic stream; $\rho^{*}$ is diminished density, to be used in the speeddensity relationships.

\subsection{Effect of MTW Lane-Sharing While Calculating the Jam Density}

From the Figs $1 \mathrm{c}$ and $1 \mathrm{~d}$ it can be seen that the number of MTWs sharing the lane with the other vehicles is increasing with the total density. Hence, the jam densities are also to be adjusted accordingly and the corresponding density diminishing factor is a characteristic of the traffic composition and the road geometry. As a general case, the jam density on a highway may be formulated as follows in the case of homogeneous traffic, without any lane-sharing behavior:

$$
\rho_{\text {jam }}=\frac{1}{L+h},
$$

where: $L$ and $h$ are respectively the length of the vehicle and the safe distance maintained by the vehicles in jam conditions.

Tang et al. (2009) improved this relation to bring in the heterogeneity of $N$ vehicle classes with $p_{j}$ as the proportion of the $j$ th vehicle class in the traffic stream:

$$
\rho_{\text {jam }}=\frac{1}{\sum_{j=1}^{N} p_{j} \cdot\left(L_{j}+h_{j}\right)},
$$

when the MTWs are present in the traffic stream along with the cars the following two cases may arise with respect to the effective jam density. 
For the traffic stream consisting of cars and MTWs:

- if all the MTWs occupy the space on the sides of the cars the jam density would be equals to or slightly greater than the density corresponding to the homogeneous traffic stream, consisting of only cars:

$\rho_{\text {jam }}^{*} \geq \frac{1}{(L+h)_{c a r}}$;

- if some of the MTWs occupy the space behind the cars, as shown in Fig. 1d, the effective jam density would be:

$$
\rho_{\text {jam }}^{*}>\frac{1}{(L+h)_{c a r}}=\frac{1}{p_{c a r s} \cdot(L+h)_{c a r s}+p_{m t w} \cdot(L+h)_{m t w}},
$$

where: $p_{\text {cars }}$ is the proportion of the cars in the traffic stream; $p_{m t w}$ is the proportion of the MTWs that are behind the cars and it is equals to zero when all the MTWs are on the sides of the cars.

In a more generalized scenario where the traffic stream consists of $N$ vehicle types, the jam density would be as follows:

$$
\rho_{j a m}^{*}=\frac{1}{\sum_{j=1}^{N-1} p_{j} \cdot\left(L_{j}+h_{j}\right)+p_{N}^{*} \cdot(L+h)_{m t w}},
$$

where: $j=1 \ldots N-1$ refers to all other vehicle types except the MTWs present in the traffic stream; $p_{j}$ refers to the corresponding proportions; $p_{N}^{*}$ is the proportion of the MTWs that are standing behind the bigger vehicles.

\subsection{Class-Specific $\Phi_{\min }$ Values}

The class specific $\Phi_{\min }$ value is the density diminishing factor corresponding to the maximum number of MTWs that can occupy the space available on the side of a particular vehicle class, under jam conditions. The diminished jam density is:

$$
\rho_{j a m}^{*}=\Phi_{\min } \cdot \rho_{j a m} .
$$

The $\Phi_{\min }$ values corresponding to each vehicle class can be used in calculating the diminished or the effective jam density. A relation to arrive at the $\Phi_{\min }$ value for a vehicle class is proposed based on the area where MTW lane-sharing takes place, i.e. the area left on the sides of that particular vehicle class:

$$
\Phi_{\min , i}=\frac{\left(L_{m t w}+h\right) \cdot\left(W_{m t w}+g\right)}{L_{i} \cdot\left(W-W_{i}\right)},
$$

where: $\Phi_{\min , i}$ is the value associated with the maximum MTW lane-sharing on the sides of class $i ; L_{m t w}$ and $W_{m t w}$ are the length and width of a typical MTW; $L_{i}$ and $W_{i}$ are the length and width of a typical $i$ th class vehicle; $h$ and $g$ are the safe longitudinal and transverse gaps, respectively, the MTW drivers would maintain at jam densities; $W$ is the width of the lane.

As an illustration, let us consider a typical case of MTW lane-sharing on the sides of the cars shown in Fig. 3.

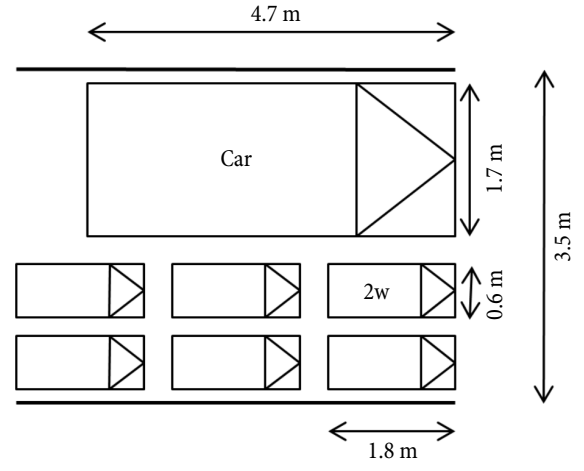

Fig. 3. MTW lane-sharing, a hypothetical case

In addition to the dimensions shown in the figure, the safe longitudinal and the transverse gaps are assumed as $0.5 \mathrm{~m}$ and $0.1 \mathrm{~m}$, respectively. In this case the value of $\Phi_{\text {min,car }}$ is 0.19 . It can be seen that the actual density is 7 vehicles over a length of $4.7 \mathrm{~m}$ or $1487 \mathrm{veh} / \mathrm{km}$, but the diminished density is 1.33 vehicles over $4.7 \mathrm{~m}$ or $283 \mathrm{veh} / \mathrm{km}$.

When several types of vehicles are present in the traffic stream and if the corresponding proportions are known the jam density may be computed as follows:

$$
\rho_{\text {jam }}^{*}=\sum_{i=1}^{N-1} \Phi_{\min , i} \cdot p_{i}+p_{N}^{*} \cdot \rho,
$$

where: $\Phi_{\min , i}$ is the minimum diminishing factor corresponding to the $i$ th vehicle class; $p_{i}$ is the corresponding vehicle's proportion in the traffic stream; $\rho$ is the actual density; $p_{N}^{*}$ is the proportion of the total number of MTWs that are behind the other vehicles.

\section{Expected Behaviour of Density Diminishing Factor from a Hypothetical Simulation Using VISSIM}

\subsection{VISSIM Simulations}

Before using the VISSIM for simulations, some of the important parameters have been adjusted for the heterogeneous traffic conditions. Though all the parameters ranging from $\mathrm{CC} 0$ to $\mathrm{CC} 9$ are approximately adjusted for the heterogeneous traffic conditions, the main emphasis was put on calibrating the time headway parameter, CC1. The model has been validated at macroscopic level using the data collected on one of the arterials located in Delhi, India. Traffic composition observed at this location is shown in Fig. 4. Important vehicle related inputs to the VISSIM are shown in Table 1. Lateral gap data for different vehicle types are shown in Table 2. It is assumed that the gaps vary linearly between the two extreme values (at zero and $60 \mathrm{~km} / \mathrm{hr}$ speeds). It was observed that the lateral gaps maintained by the vehicles when traveling at different speeds have significant influence on the output from VISSIM. Finally the observed and the simulated flow-occupancy relations are shown in Fig. 5. From the comparison of observed and simulated flow-occupancy relationships it can be said that VISSIM is able to simulate the heterogeneous traffic conditions with reasonable accuracy. 


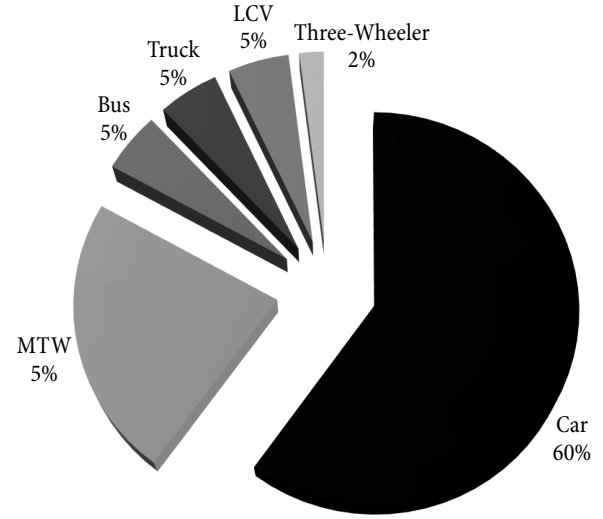

Fig. 4. Traffic composition used for validating VISSIM

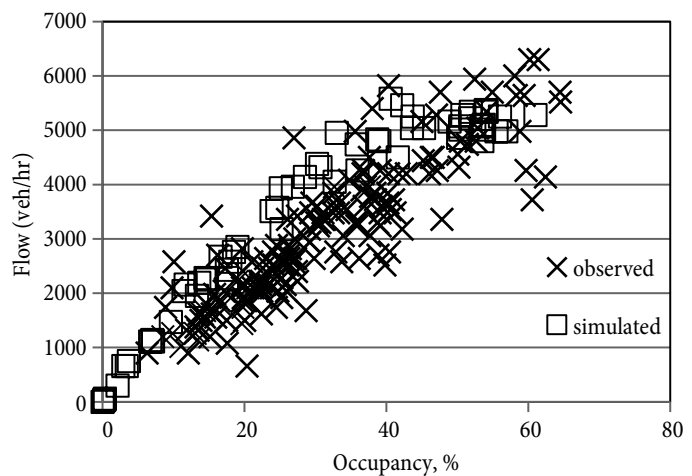

Fig. 5. Observed and simulated flow-occupancy relationship

Table 1. Acceleration deceleration behavior of various types of vehicles used in VISSIM validation

\begin{tabular}{lcccc}
\hline \multirow{2}{*}{ Vehicle type } & \multicolumn{3}{c}{ Acceleration $\left[\mathrm{m} / \mathrm{sec}^{2}\right]$ at different speeds } & Deceleration $\left[\mathrm{m} / \mathrm{sec}^{2}\right]$ \\
\cline { 2 - 5 } & $\leq 5.5 \mathrm{~m} / \mathrm{sec}$ & Between 5.5 and $11 \mathrm{~m} / \mathrm{sec}$ & 1.0 & 2.0 \\
\hline Car & 2.0 & 1.5 & 1.5 & 1.5 \\
\hline MTW & 2.5 & 2.0 & 0.5 & 1.5 \\
\hline Bus & 1.5 & 1.0 & 0.5 & 1.5 \\
\hline Truck & 1.0 & 0.5 & 0.5 & 1.5 \\
\hline LCV & 1.0 & 1.0 & 0.5 & 1.5 \\
\hline Three-Wheeler & 1.0 & 1.0 & & 1.5 \\
\hline
\end{tabular}

Table 2. Gaps maintained by various types of vehicles at 0 and $60 \mathrm{~km} / \mathrm{hr}$ speed (Nagaraj et al. 1990)

\begin{tabular}{lcc}
\hline Type of vehicle & $\begin{array}{c}\text { Lateral gap } \\
\text { at zero speed }[\mathrm{m}]\end{array}$ & $\begin{array}{c}\text { Lateral gap } \\
\text { at }\end{array}$ \\
\hline Bus & 0.4 & 1.0 \\
\hline Truck & 0.4 & 1.0 \\
\hline $\begin{array}{l}\text { Light Commercial } \\
\text { Vehicle }\end{array}$ & 0.3 & 0.7 \\
\hline Car & 0.3 & 0.7 \\
\hline MTW & 0.1 & 0.7 \\
\hline Three-Wheeler & 0.2 & 0.7 \\
\hline
\end{tabular}

\subsection{Results from VISSIM}

The density diminishing factor $\Phi$ is expected to exhibit inverse proportionality behavior with the total density of the stream as well as with the relative density of the MTWs. Same was evident from the simulation of a hypothetical single lane road stretch using VISSIM. VISSIM is based on Wiedemann's car-following model and does not account for the MTW lane-sharing behavior. However, VISSIM is capable of simulating the overtaking behavior within a lane if the available lateral gap is sufficient. As shown in Fig. 2, overtaking is a phenomenon that is comparable to the side-by-side movement. The major difference is that the overtaking operation is completed in a considerably lower amount of time compared to the side-by-side movement. Same is evident from the simulation of a hypothetical single lane stretch allowing and prohibiting the within-lane overtaking. Output from this simulation is used in observing the trend of the density diminishing factor with respect to the total density and the fraction of MTWs. The trend of the density diminishing factor is obtained from the comparisons of the speed-density relations corresponding to the scenarios, overtaking permitted, and overtaking prohibited. Several simulation runs have been carried out for different MTW proportions to know the relationship between the fraction of MTWs and the density diminishing factor.

The density and the avg. speed data were obtained directly from VISSIM. The data from VISSIM are averaged over one-minute duration. As the simulations are carried out for one hour period, sixty data points on the density and the speed have been obtained. Speed-density relations obtained from the simulation runs, for different fractions of MTWs, are shown in Fig. 6. The density values with overtaking permitted are always greater than those with overtaking prohibited, corresponding to the same stream speed. It may be observed that irrespective of the MTW and four-wheeler proportions, the difference in densities increases with the increasing density. Further, magnitude of the difference in densities increases with the increase in the proportion of the MTWs. Variation of density diminishing factor with speed, for various proportions of MTWs, is shown in Fig. 7. From this figure it can be seen that the fraction of MTWs present in the traffic stream significantly influence the density diminishing factor. At the same time for from 40 to $60 \%$ of MTWs there is not much change 
in the diminishing factor. More simulations, with varying proportions of MTWs, may give a better insight into this behavior. Fig. 8 shows the variability of threshold speed with respect to the fraction of MTWs observed in the traffic stream. Threshold speed for lane-sharing is $65 \mathrm{~km} / \mathrm{hr}$ for the MTW proportion ranging from 25 to $60 \%$. When $90 \%$ of MTWs are present in the traffic stream lane-sharing is observed even at higher speeds.

Correlating the behavior found in the cases of overtaking permitted and overtaking prohibited to the conditions with and without MTW lane-sharing, it may be concluded that the density diminishing factor $\Phi$ is inversely proportional to the total density as well as to the relative density of the MTWs (Fig. 7). a)

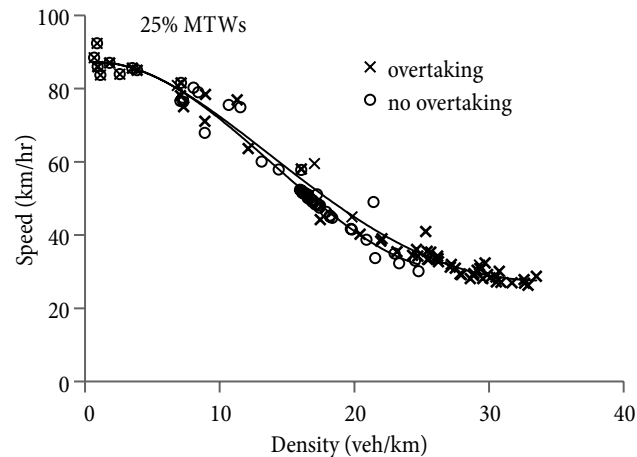

c)

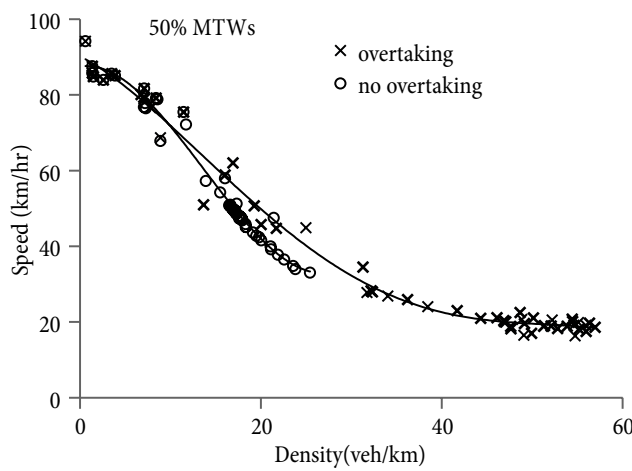

e)

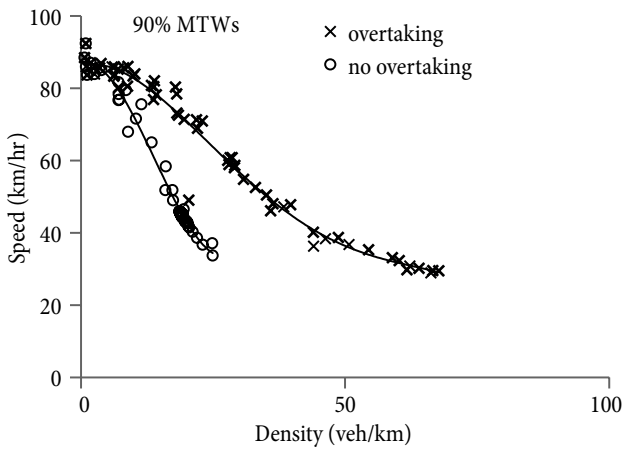

b)

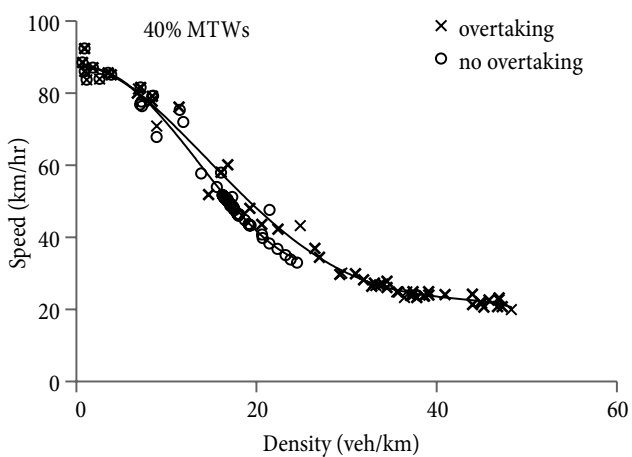

d)

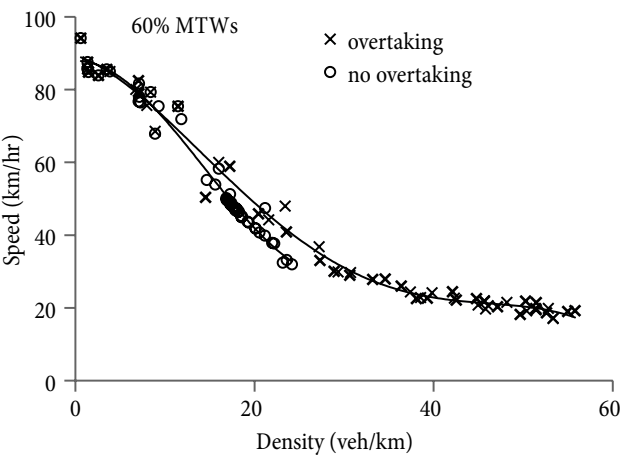

Fig. 6. Flow-density curves at: a - 25\% MTW, rest all being cars; b - 40\% MTW, rest all being cars; c - 50\% MTW, rest all being cars; d - 60\% MTW, rest all being cars; e - 90\% MTW, rest all being cars

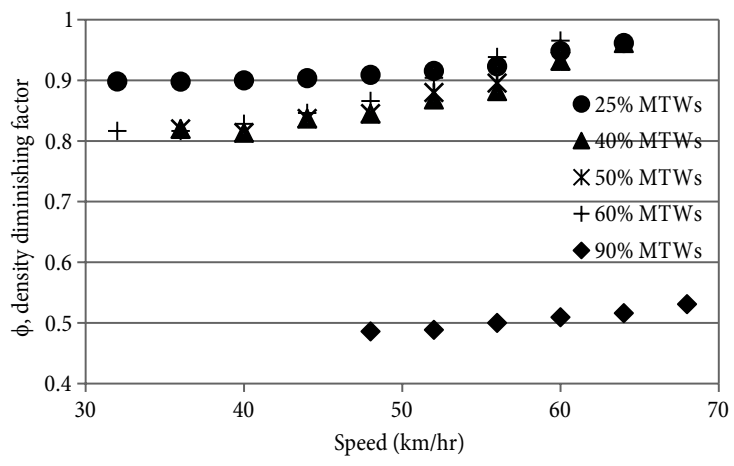

Fig. 7. Variation of density diminishing factor with speed for various proportions of MTWs

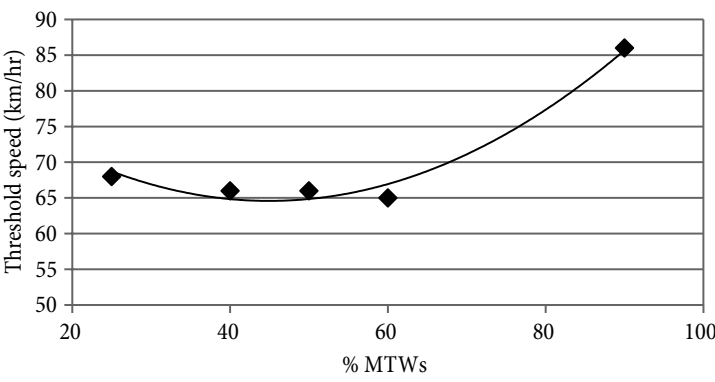

Fig. 8. Variation of threshold speed for lane-sharing with the proportion of MTWs 
The difference in densities corresponding to the same average traffic stream speed, as can be seen in Fig. 6, is attributed to the side-by-side movement of the MTWs within a single lane. Hence when the speeddensity relation is given as the input to the macroscopic traffic flow models the difference in the densities must be reflected in that relation.

\section{Summary and Conclusions}

Lane-sharing behavior of the MTWs is a crucial aspect to be analyzed when modeling the heterogeneous urban traffic observed in the developing countries like India. Lane-sharing behavior of the MTWs significantly influences the applicability of microscopic as well as the macroscopic traffic characteristics. Headways and the corresponding macroscopic characteristics do not describe the actual traffic behavior when there is no lane discipline along with the lane-sharing of the MTWs. It is necessary to integrate the lane-sharing behavior with the traffic characteristics before using them in the modeling process. In this study a factor termed as 'the density diminishing factor' has been proposed to compute the effective density. Density diminishing factor considers the number of MTWs that are using the road space available adjacent to the other vehicles.

Speed, as a function of the density, is a crucial input to the macroscopic traffic flow models. Speed-density relations have been obtained for the lane disciplined and no-lane disciplined traffic streams using the microscopic simulation software VISSIM. No lane-discipline has been regenerated by allowing the overtaking/passing within the same lane. From the resulting speed-density relations it has been found that the density diminishing factor will be inversely proportional to the total density as well as to the relative density of the MTWs. It has also been observed that the density diminishing factor is found to be significantly influenced by the proportions of MTWs present in the traffic stream. From the limited simulations, it has been observed that the lane-sharing behavior starts taking place when the average stream speed is less than $65 \mathrm{~km} / \mathrm{hr}$, for the proportions of MTWs in between 25 to $60 \%$. For further higher MTW proportions lane-sharing has been observed even in free flow conditions. More field observations along with several simulation runs are planned in further research for better understanding of the density diminishing factor.

\section{References}

Bhavathrathan, B. K.; Mallikarjuna, C. 2012. Evolution of macroscopic models for modeling the heterogeneous traffic: an Indian perspective, Transportation Letters 4(1): 29-39. http://dx.doi.org/10.3328/TL.2012.04.01.29-39

Del Castillo, J. M.; Benítez, F. G. 1995. On the functional form of the speed-density relationship - I: general theory, Transportation Research Part B: Methodological 29(5): 373-389. http://dx.doi.org/10.1016/0191-2615(95)00008-2

Gupta, A. K.; Katiyar, V. K. 2007. A new multi-class continuum model for traffic flow, Transportmetrica 3(1): 73-85. http://dx.doi.org/10.1080/18128600708685665

Haque, M. M.; Chin, H. C.; Huang, H. 2008. Examining exposure of motorcycles at signalized intersections, Transportation Research Record 2048: 60-65.

http://dx.doi.org/10.3141/2048-08
Khan, S. I.; Maini, P. 1999. Modeling heterogeneous traffic flow, Transportation Research Record 1678: 234-341. http://dx.doi.org/10.3141/1678-28

Lan, L. W.; Chiou, Y.-C.; Lin, Z.-S.; Hsu, C.-C. 2010. Cellular automaton simulations for mixed traffic with erratic motorcycles' behaviours, Physica A: Statistical Mechanics and its Applications 389(10): 2077-2089. http://dx.doi.org/10.1016/j.physa.2010.01.028

Lee, T.-C.; Polak, J. W.; Bell, M. G. H. 2009. New approach to modeling mixed traffic containing motorcycles in urban areas, Transportation Research Record 2140: 195-205. http://dx.doi.org/10.3141/2140-22

Logghe, S.; Immers, L. H. 2008. Multi-class kinematic wave theory of traffic flow, Transportation Research Part B: Methodological 42(6): 523-541. http://dx.doi.org/10.1016/j.trb.2007.11.001

Mallikarjuna, C.; Ramachandra Rao, K. 2009. Cellular automata model for heterogeneous traffic, Journal of Advanced Transportation 43(3): 321-345. http://dx.doi.org/10.1002/atr.5670430305

Meng, J.-P.; Dai, S.-Q.; Dong, L.-Y.; Zhang, J.-F. 2007. Cellular automaton model for mixed traffic flow with motorcycles, Physica A: Statistical Mechanics and its Applications 380: 470-480. http://dx.doi.org/10.1016/j.physa.2007.02.091

Minh, C. C.; Sano, K.; Matsumoto, S. 2005. The speed, flow and headway analyses of motorcycle traffic, Journal of the Eastern Asia Society for Transportation Studies 6: 1496-1508.

Nagaraj, B. N.; George, K. J.; John, P. K. 1990. A study on linear and lateral placement of vehicles in mixed traffic environment through video-recording, Highway Research Bulletin 42: 105-135.

Ngoduy, D.; Liu, R. 2007. Multiclass first-order simulation model to explain non-linear traffic phenomena, Physica A: Statistical Mechanics and its Applications 385(2): 667-682. http://dx.doi.org/10.1016/j.physa.2007.07.041

Powell, M. 2000. A model to represent motorcycle behaviour at signalised intersections incorporating an amended first order macroscopic approach, Transportation Research Part A: Policy and Practice 34(7): 497-514. http://dx.doi.org/10.1016/S0965-8564(99)00027-0

Rongviriyapanich, T.; Suppattrakul, C. 2005. Effects of motorcycles on traffic operations on arterial streets, Journal of the Eastern Asia Society for Transportation Studies 6: 137-146.

Su, J.-M.; Wu, C.-H. E.; Hung, C.-Y. 2006. Traffic characteristics of motorcycles on tangent sections of expressways, Transportation Research Record 1969: 126-133. http://dx.doi.org/10.3141/1969-20

Tang, T. Q.; Huang, H. J.; Shang, H. Y. 2011. A macro model for bicycle flow and pedestrian flow with the consideration of the honk effects, International Journal of Modern Physics B: Condensed Matter Physics; Statistical Physics; Atomic, Molecular and Optical Physics 25(32): 4471-4479. http://dx.doi.org/10.1142/S0217979211059462

Tang, T.-Q.; Huang, H.-J.; Shang, H. Y. 2010. A dynamic model for the heterogeneous traffic flow consisting of car, bicycle and pedestrian, International Journal of Modern Physics C: Computational Physics and Physical Computation 21(2): 159-176. http://dx.doi.org/10.1142/S0129183110015038

Tang, T. Q.; Huang, H. J.; Zhao, S. G.; Shang, H. Y. 2009. A new dynamic model for heterogeneous traffic flow, Physics Letters A 373(29): 2461-2466. http://dx.doi.org/10.1016/j.physleta.2009.05.006

Wong, G. C. K.; Wong, S. C. 2002. A multi-class traffic flow model - an extension of LWR model with heterogeneous drivers, Transportation Research Part A: Policy and Practice 36(9): 827-841.

http://dx.doi.org/10.1016/S0965-8564(01)00042-8 